\title{
Molecular clone and functional study of a novel hepatoma associated gene
}

\author{
YANHONG LIU, YANJIE ZHAO, QIANG JU, LIN CHEN, FENG LI, \\ GUOHUA ZHOU, PINGLI XIE, GUANCHENG LI and YUEHUI LI
}

\begin{abstract}
Tumor Immunobiology Laboratory of Cancer Research Institute, Key Laboratory of Carcinogenesis and
Cancer Invasion, Ministry of Education, Key Laboratory of Carcinogenesis, Ministry of Health,

Central South University, Changsha, Hunan 410078, P.R. China
\end{abstract}

Received October 28, 2012; Accepted December 19, 2012

DOI: $10.3892 /$ ijo.2013.1766

\begin{abstract}
HTA is a novel hepatoma associated gene screened by bioinformatic strategies in our previous study. In the present investigation, the full-length sequence of the HTA gene was cloned by 3'-rapid amplification of cDNA ends (RACE) and 5'-RACE, which was $1414 \mathrm{bp}$ and the open reading frame (ORF) was constituted with 3 exons and 2 introns. There are two types of splicing of the mRNA of HTA. Northern blot analysis showed that the $1.4 \mathrm{~kb} H T A$ mRNA and $1.7 \mathrm{~kb} H T A$ mRNA transcripts were present in the hepatocellular carcinoma (HCC) cell lines HepG2 and QGY-7703 but not in the normal cell line L02 and the human umbilical vein endothelial cells (HUVECs). Overexpression of the HTA gene in the hepatic cell line QSG-7701 via stable transfection can promote its proliferation rate and colony forming ability and change the cell cycle distribution of the cell lines. These results showed that the HTA gene is a potential therapeutic target in HCC and the clarification of its gene structure and sequence information provide an essential tool for future research.
\end{abstract}

\section{Introduction}

Hepatocellular carcinoma (HCC) is a highly aggressive cancer with multiple causes and no currently available effective treatment $(1,2)$. Understanding the molecular mechanism of HCC carcinogenesis, its development and progression is imperative for developing novel, effective and targeted therapies for this fatal disease $(3,4)$. Numerous investigations using different

Correspondence to: Professor Guancheng Li or Dr Yuehui Li, Tumor Immunobiology Laboratory of Cancer Research Institute, Central South University, 110 Xiangya Road, Changsha, Hunan 410078, P.R. China

E-mail: libsun@163.com

E-mail: yuehuili7927@yahoo.com.cn

Key words: hepatoma associated gene, full length cDNA, rapid amplification of cDNA ends, northern blot analysis, overexpression, cell proliferation improved approaches, such as insertional mutagenesis screen (5), in vivo RNAi screen (6) and omics-based methods (7), are being conducted. Novel tumor associated genes such as glypican-3 (GPC3), astrocyte elevated gene-1 (AEG-1) and exportin 4 (XPO4) have been discovered and studied extensively $(8,9)$.

To search for new biomarkers for tumor diagnosis and therapy, we previously detected tumor associated genes using bioinformatic approaches on a large scale. HTA is a novel tumor associated gene screened by $c D N A x$ Profile, an in silico identification tool based on the EST database provided by the Cancer Genome Anatomy Project (CGAP) web site and preliminarily confirmed by electronic-northern (E-northern). RT-PCR revealed that HTA (NM 001101347) was specifically expressed in certain types of tumor and had a high expression rate in HCC. To further elucidate its mechanism in hepatoma carcinogenesis and its potential role as a cancer biomarker, research were carried out and the results showed that knockdown of endogenous HTA expression in the malignant hepatic cell line HepG2 by small interfering RNA (siRNA) attenuated cell growth, weakened its tumor formation ability in nude mice (10) and changed the expression level of some genes associated with apoptosis (Liu et al, unpublished data), which suggests that HTA may be important in HCC development and progression and its mechanism might be apoptosis-related.

Since HTA is a gene screened from the EST database, the full length sequences were essential for its functional investigation. In this report, the full length cDNA of the HTA gene was verified by rapid amplification of cDNA 3'-ends [3'-rapid amplification of cDNA ends (RACE)] and amplification of cDNA 5'-ends (5'-RACE) $(11,12)$. Northern blot assay was performed to determine the transcripts of HTA mRNA expression in HCC and normal hepatic cell lines.

\section{Materials and methods}

Cell lines. The HepG2, QGY-7703, HUVEC, L-02, QGY-7701 cell lines were used in this study. The malignant hepatic cell line HepG2 was stored by our laboratory. QGY-7703 was obtained from the cell bank of the Chinese Academy of Sciences, Shanghai, China. The benign hepatic cell lines QSG-7701, L-02 were stored by our laboratory. Human 
umbilical vein endothelial cells (HUVEC) were stored by our laboratory. These cell lines were cultured in Gibco ${ }^{\circledR}$ RPMI1640 medium, supplemented with $10 \%$ FBS. All cells were maintained at $37^{\circ} \mathrm{C}$ in a $5 \% \mathrm{CO}_{2}$ incubator.

Preparation of RNA. Total RNA from cell lines was extracted using TRIzol RNA isolation reagents (Life Technologies) according to the manufacturer's instructions. The mRNA was prepared from total RNA using the PolyATtract ${ }^{\circledR}$ mRNA Isolation Systems (Promega) according to the manufacturer's instructions. All the RNA samples were tested for integrity and purity by an ultraviolet spectrophotometer (OD260/OD280), RNA temperature incubation experiment and formaldehyde degeneration electrophoresis.

Cloning and sequencing of full length HTA cDNA. In accordance with the existing sequence of the HTA gene obtained in a previous study (10), the gene specific primers for 5'-RACE (GSP1), 3'-RACE (GSP2) and for nest PCR NGSP1 were designed as shown in Fig. 1 and Table I. Then, the 5'-end sequence and 3 '-end sequence of the cDNA encoding the HTA gene were obtained from the HCC cell line HepG2 using Smarter ${ }^{\mathrm{TM}}$ RACE cDNA Amplification kit 5'/3' (Clontech) according to the manufacturer's instructions. Initially with $1 \mu \mathrm{g}$ total RNA of HepG2 cells, the 3'-RACE ready cDNA and 5'-RACE ready cDNA were synthesized according to the protocol, respectively. GSP1 primer and Upper primer mix were then used to amplify the 5'-end sequences of the HTA gene. Upper primer mix and GSP2 primer were used to amplify the 3 '-end sequence of the HTA gene. Then, a touch-down program was performed as follows: 5 cycles of denaturing at $94^{\circ} \mathrm{C}$ for $30 \mathrm{sec}, 72^{\circ} \mathrm{C}$ for $3 \mathrm{~min}$, followed by 5 cycles of each $94^{\circ} \mathrm{C}$ for $30 \mathrm{sec}, 70^{\circ} \mathrm{C}$ for $30 \mathrm{sec}$ and $72^{\circ} \mathrm{C}$ for $3 \mathrm{~min}$, then followed by 35 cycles of $94^{\circ} \mathrm{C}$ for $30 \mathrm{sec}, 68^{\circ} \mathrm{C}$ for $30 \mathrm{sec}$ and $72^{\circ} \mathrm{C}$ for $3 \mathrm{~min}$. The RACE PCR product was analyzed by $1 \%$ agarose gel electrophoresis and photographed by Image Master VDS Gel Imaging system (Pharmacia). Using 25-fold diluted RACE PCR product as template, nest PCR was performed by nest primers and program as follows: 30 cycles of $94^{\circ} \mathrm{C}$ for $30 \mathrm{sec}$, $68^{\circ} \mathrm{C}$ for $30 \mathrm{sec}$ and $72^{\circ} \mathrm{C}$ for $3 \mathrm{~min}$. The nest PCR product was separated by $1 \%$ low-melting agarose gel electrophoresis and Objective band was retrieved and ligated to PGEM-T easy vector, then transformed into Escherichia coli (E. coli) DH5a. Several positive clones were picked out and sequenced.

Analysis of HTA gene structure and alternative splicing. The sequences of products of RACE PCR positive clones were comprehensively analyzed by BLAST (http://www. ncbi.nlm.nih.gov/BLAST/) and online software open reading frame (ORF) finder (http://www.ncbi.nlm.nih.gov/gorf/orfig. cgi) provided by the National Center For Biotechnology Information (NCBI). The full length sequence primers of HTA (HTA-cDNA-F and HTA cDNA-R in Table I) were designed and the full lengths of $H T A$ transcripts were amplified. The amplified product was sequenced and the alternative splicing of the HTA gene was analyzed.

Northern blot analysis of HTA mRNA expression. Probes for northern blotting were produced using a DIG RNA labeling and detection kit (Roche Diagnostics). A 589-bp HTA gene specific primer pair was designed for amplification of northern bolt probe; at the same time, a 24-bp T7 promoter sequence 5'-CTAATACGACTCACTATAGGGAGA-3' was added to the 5 '-end of reverse primer (Table I). Then, the northern blot probe of HTA gene labeling with digoxin in UTP was produced by in vitro transcription. GAPDH probe was produced with the same procedure. The labeling efficiency of the probes was determined according to the manufacturer's instructions.

mRNA $(1 \mu \mathrm{g})$ from four hepatic cell lines (HUVEC, L-02, HepG2, QGY-7703) was separated on denaturing 0.667\% formaldehyde- $2 \%$ agarose gel and was transferred onto positively charged nylon membrane Biodyne Plus (Pall) by the capillary transfer method. Following UV cross-linking (245 nm, $1.5 \mathrm{~J} / \mathrm{cm}^{2}$ for $1 \mathrm{~min} 45 \mathrm{sec}$ ), prehybridization was carried out at $68^{\circ} \mathrm{C}$ for $1 \mathrm{~h}$ using DIG Easy Hyb (Roche Diagnostics). Hybridization was performed at $68^{\circ} \mathrm{C}$ for $12 \mathrm{~h}$, 40 revolutions per minute (rpm), using the DIG Easy Hyb containing $100 \mathrm{ng} / \mathrm{ml}$ of the complementary RNA (cRNA) probe that was previously produced. The membrane was then washed twice for $5 \mathrm{~min}$ in $2 \mathrm{X} \mathrm{SSC}$ with $0.1 \%$ sodium dodecyl sulfate (SDS) at room temperature, and was washed again twice in $0.1 \mathrm{X}$ SSC with $0.1 \%$ SDS at $68^{\circ} \mathrm{C}$ for $15 \mathrm{~min}$. After incubating the membrane in alkaline phosphatase (AP) labeled anti-digoxin antibody, the chemiluminescent signals were detected using the DIG RNA labeling and detection kit according to the manufacturer's instructions. Signals were detected after exposure on Pierce ECL Plus (Thermo Scientific). For standardization, the HTA probe was stripped from the membrane and was reprobed using the DIG-labeled GAPDH RNA probe under conditions similar to assessing the RNA loading and transfer efficiency.

Construction of expression vectors of HTA. Total RNA was extracted from HepG2 cells by homogenization in TRIzol reagent (Invitrogen, USA) and first-strand cDNAs were synthesized from $2 \mu \mathrm{g}$ of DNase I-treated total RNA using First Strand cDNA Synthesis kit (Fermentas, USA) by way of a two-step system. Gene-specific polymerase chain reaction (PCR) primers for HTA ORF amplification were: 5'-CGC GGATCCGCCGCCATGCTCTGTGTGCCCTTCCT-3' (sense) and 5'-CCGGAATTCTTAGCCCAAGATGAAGACAA GGC-3' (antisense) and introduced with BamHI site (sense) and an EcoRI site (antisense) respectively (underlined). The italic $G C C G C C$ in the sense primer was a Kozak sequence (13) designed to improve gene expression quantity. The bold ATG and TAA were initial and terminator codon, respectively. The PCR products of the HTA gene were purified by $1 \%$ agarose gel electrophoresis then double digested and ligated into the eukaryotic expression vector pcDNA3.1(+). The recombinant plasmid was transformed into E. coli $D H 5 \alpha$ then identified by PCR, double endonuclease digestion and DNA sequencing.

Transfection and selection of stable HTA overexpression hepatic cell line. Plasmids pcDNA3.1(+)-HTA and pcDNA3.1(+) were transfected into QSG-7701 cell lines using Lipofectamine $^{\mathrm{TM}} 2000$ (Invitrogen). Three days later, cells were selected in a medium containing $G 418(600 \mathrm{mg} / \mathrm{ml})$ for 10 days, then the limiting dilution method was used and G418-resistant colonies were cloned and expanded. RT-PCR 
Table I. Primers used in this study.

Primer

Universal primer (long)

Universal primer (short)

Nested universal primer

GSP1

GSP2

NGSP1

HTA-cDNA-F

$H T A-\mathrm{cDNA}-\mathrm{R}$

HTA-probe-F

HTA-probe-R-T7

GAPDH-probe-F

GAPDH-probe-R-T7
CTAATACGACTCACTATAGGGCAAGCAGTGGTATCAACGCAGAGT CTAATACGACTCACTATAGGGC

AAGCAGTGGTATCAACGCAGAGT

TCTGCTGGAGGTGAGGGCTCGTGGT

ATGGGTTGCTTGGGGCATCCTGTG

GTCGGGGTTGTTCTTGTTTTCAG

CGAAGATTTCTTATAGACAGGC

GGAGGGCATTATGGTGAG

CTATTTATGGGTTGCTTGG

CTAATACGACTCACTATAGGGAGAGAGGGCATTATGGTGAGAT

AATCCCATCACCATCTTCC

CTAATACGACTCACTATAGGGAGAGCTGTAGCCAAATTCGTTGT

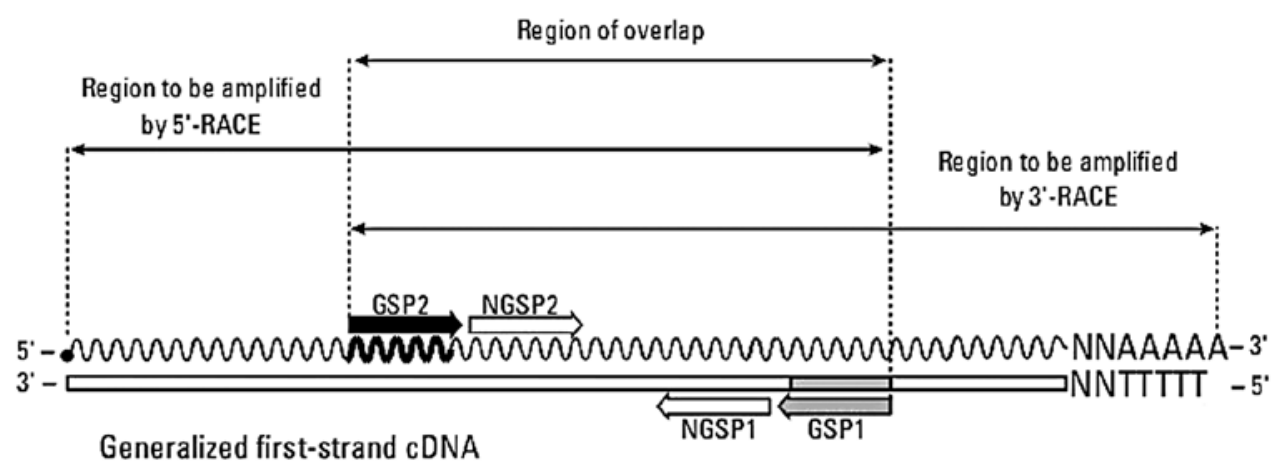

Figure 1. The location and direction of primers for 3'/5'-RACE.

was performed to detect the HTA mRNA expression level in empty vector and HTA overexpression clones. A clone with the highest HTA expression was selected to perform subsequent experiments.

Cell proliferation test in vitro. Growth experiments of QSG-7701-c [QSG-7701 cell line stably transfected with pcDNA3.1 (+) empty vector as a control] and QSG-7701-HTA-2 were performed using the Cell Counting kit-8 (CCK-8, Beyotime) based on WST-8 [2-(2-methoxy-4-nitrophenyl)-3(4-nitrophenyl)-5-(2,4-disulfophenyl)-2H-tetrazolium]. Cell doubling time test and plate cloning formation assay were reconfirmed by cell cycle analysis, which was performed by flow cytometry.

In the CCK-8 assay, cells were seeded with serum-free medium at a density of $10^{3}$ cells/well in 96-well plates $(n=6)$, grown overnight, washed with PBS, and incubated in DMEM medium supplemented with 5\% FBS. The samples were tested every $24 \mathrm{~h}$ for 6 days. WST-8 (10 $\mu \mathrm{l})$ each well was added for $2 \mathrm{~h}$ and the optical density was measured at $450 \mathrm{~nm}$ in an SM-3 automatic enzyme-linked immuneanalyzer (TianShi, China).

In the cell doubling time test, cells were seeded at a density of $10^{4}$ cells/well in 24 -well plates $(n=3)$, grown with serum- free medium overnight, washed with PBS, and incubated in DMEM medium with 3\% FBS. Cells were collected 6 days later and cell densities were assessed by counting the cells in a hemocytometer. Cell doubling time $\left(T_{D}\right)$ was calculated with the following formula: $T_{D}=t[\lg 2 /(\lg N t-\lg N o)][t$, period of culturing (hour). No, cell density when the cells were seeded. $N t$, cell density when the cells were cultured $t$ hours].

Plate colony formation assay was used to assess the colony formation ability of cells. The cells were incubated in serumfree medium overnight. A total of 1,000 single-cell suspension cells resuspended in $2 \mathrm{ml}$ growth media were plated in triplicate on 6 -well plates. Colonies $>50$ cells were counted 14 days after plating. Colony formation ratio was calculated with the following formula: Colony formation rate $=$ (number of colonies counted /numbers of cells seeded) x $100 \%$.

For flow cytometry, cells were incubated with serum-free medium for $24 \mathrm{~h}$, and then cultured in DMEM with $10 \%$ FBS for $48 \mathrm{~h}(\mathrm{n}=3)$. Cells were harvested and resuspended in fixation fluid at a density of $10^{6} / \mathrm{ml}, 1,500 \mu \mathrm{l}$ propidium iodide (PI) solution was added, and the cell cycle was detected by FACSCalibur (Becton-Dickinson).

Statistical analysis. The values are presented as the means \pm SD for three or more individual experiments. Data were tested 


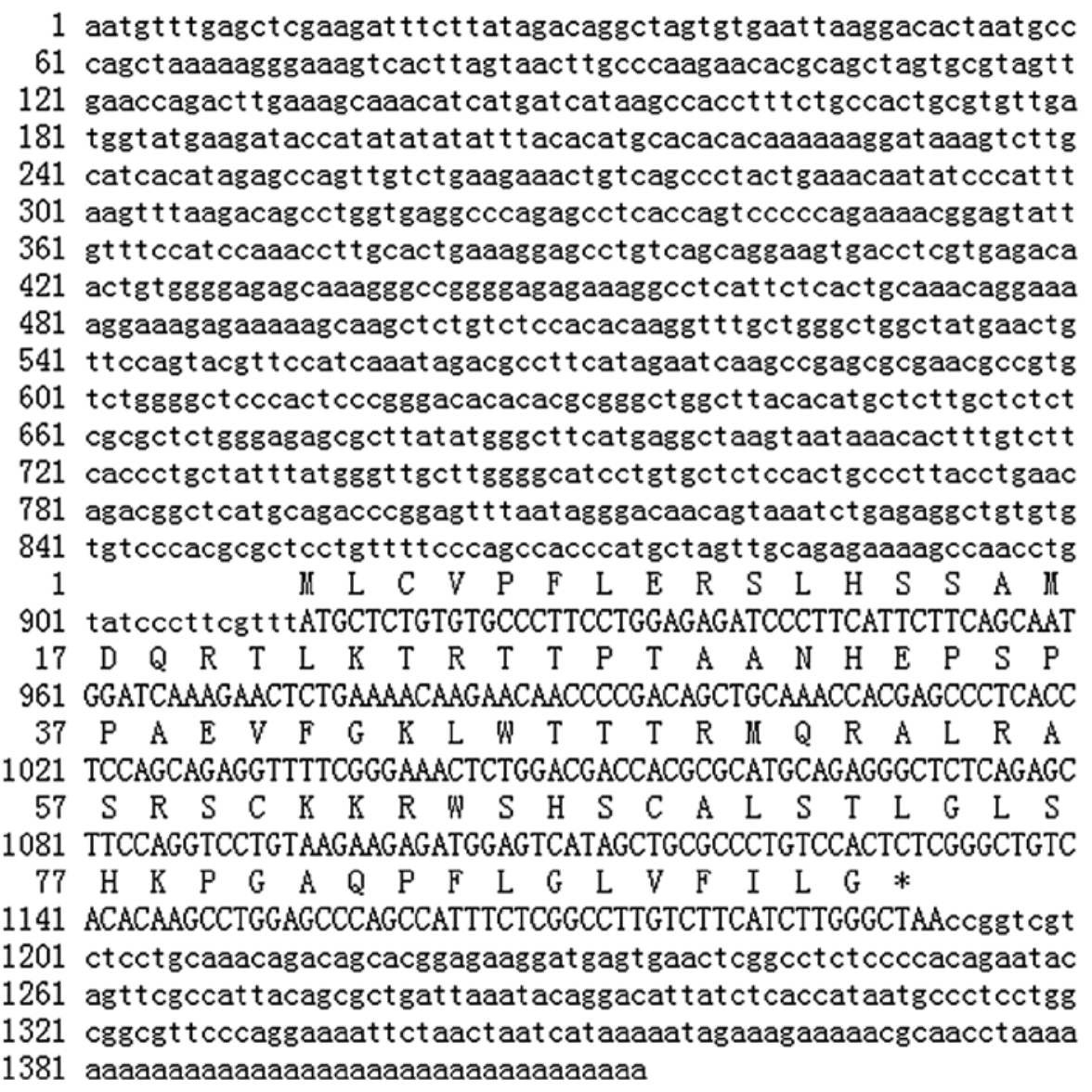

Figure 2. The full length sequence and the deduced amino acid sequence of the HTA gene. with SPSS software (version 13.0, SPSS Inc.) for significance. The analysis of variance and t-test were applied in comparing the intergroup difference of measurement data. $p<0.05$ was considered to indicate a statistically significant difference. The diagrams were drawn by GraphPad Prism 5.

\section{Results}

Molecular cloning of the full-length HTA cDNA. We cloned the 5'-RACE and 3'-RACE product and determined the sequence. The products using this method were partly consistent with a previous known sequence of HTA. Finally, the 1414-bp obtained HTA cDNA was reconfirmed to be full length by northern blot analysis. Fig. 2 shows the nucleotide sequence and the deduced amino acid sequence using 5'-RACE and 3'-RACE. The 3'-end of the product was in line with the sequence previously obtained. The ORF of the HTA gene encoded a protein of 92 amino acids with an estimated molecular weight of $10.2 \mathrm{kDa}$. A comparison of the HTA cDNA sequence with the GenBank database showed no significant homology with other genes except its homologous sequences in Macaca fascicularis and Macaca fascicularis genome. Furthermore, a comparison of the deduced amino acid sequence with a protein sequence database showed no overall significant homology with other proteins whose function is known. It also showed no conserved domains and no valid domain hit for architecture searched by NCBI.

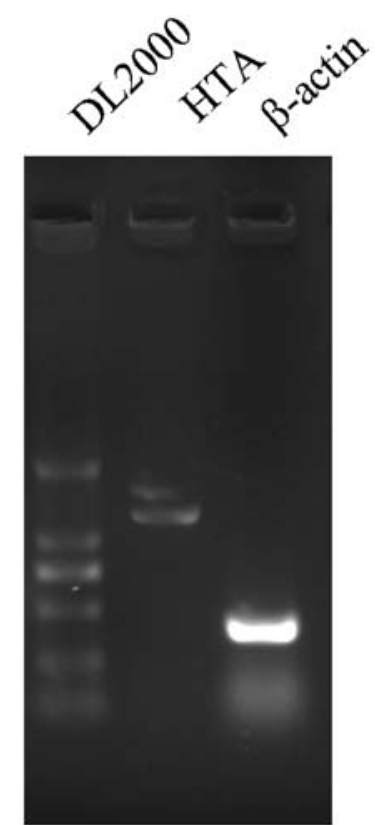

Figure 3. Full length amplification of the HTA gene.

Alternative splicing analysis of the HTA gene. Online prediction software ORF finder provided by NCBI revealed that the HTA gene was constituted with 3 exons and 2 introns. RT-PCR with HTA full length primer pair HTA-cDNA-F and 
A
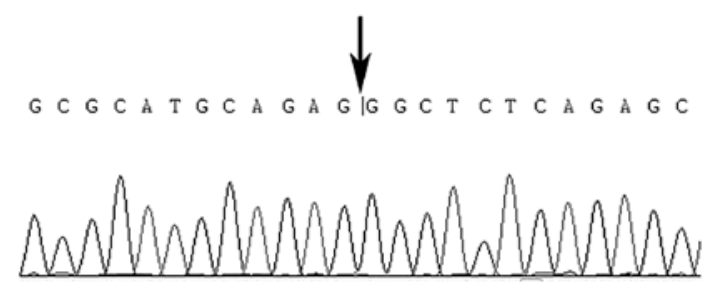

B
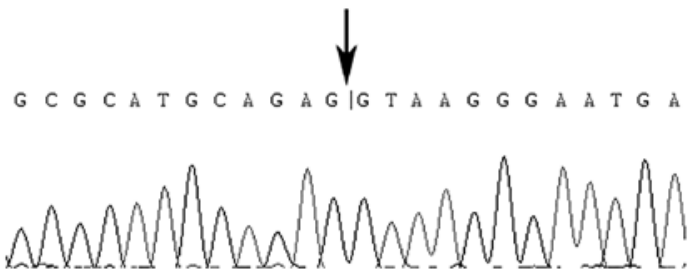

C

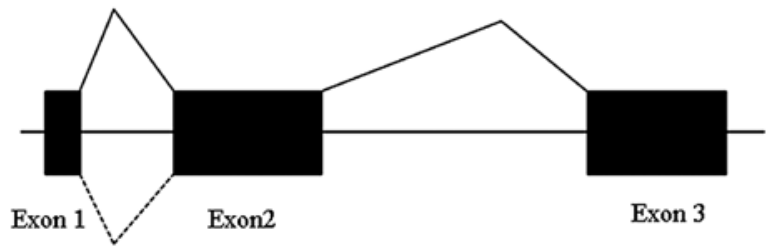

Figure 4. Alternative splicing of the HTA gene. (A) Sequence of the $1.4 \mathrm{~kb}$ transcript; (B) sequence of the $1.7 \mathrm{~kb}$ transcript; (C) sketch map of alternative splicing form.

A

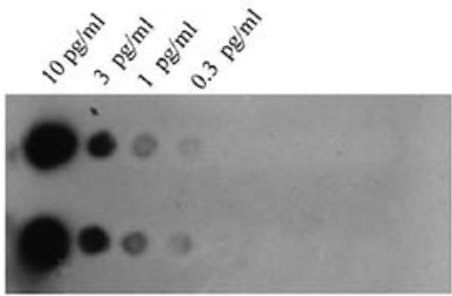

HTA

GAPDH

B

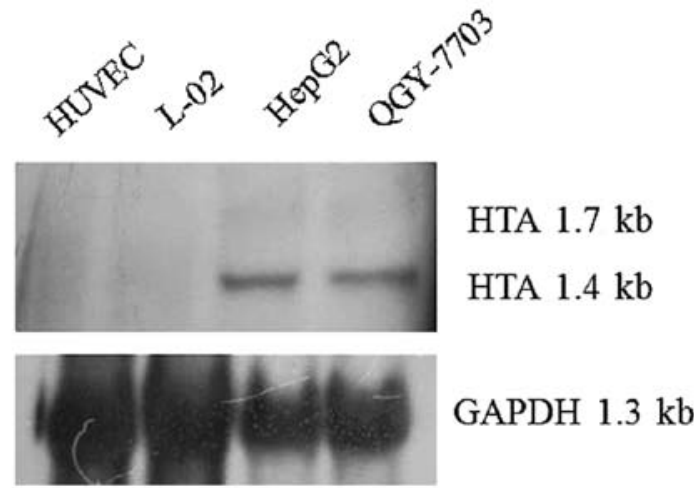

Figure 5. Expression of HTA in different hepatic cell lines detected by northern blot analysis. (A) Labeling efficiency of HTA and GAPDH digoxin probes. (B) Expression of HTA and GAPDH.

HTA-cDNA-R showed that the HTA gene has two transcripts which were 1.4 and $1.7 \mathrm{~kb}$, respectively (Fig. 3). Sequencing of RT-PCR product suggested an alternative splicing of the HTA gene. The 252-bp second introns of the HTA gene can be spliced, or retained as a part of mRNA (Fig. 4).

Expression of HTA mRNA in hepatic cell lines. The labeling efficiency of HTA and GAPDH digoxin probes were defined.
When the samples were diluted to the concentration of $0.3 \mathrm{pg} / \mu \mathrm{l}$, the signal could still be detected, which means that adequate amounts of labeled RNA probe for northern blot analysis were obtained (Fig. 5A). Northern blot analysis showed that the 1.4-kb HTA mRNA and 1.7-kb HTA mRNA transcripts were present in HCC cell lines HepG2 and QGY-7703. The expression amount of the 1.4-kb transcript was much higher than the $1.7-\mathrm{kb}$ transcript. In the normal hepatic cell line L-02 and the normal cell line HUVEC, no HTA transcript was detected (Fig. 5B).

Establishment of the overexpression of the HTA hepatic cell line. The recombinant eukaryotic expression plasmid pcDNA3.1(+)-HTA was successfully constructed and verified by bacterial colony PCR, restriction enzyme digestions (Fig. 6A) and complete sequencing (Fig. 6B). The recombinant HTA protein obtained from this plasmid had a Kozak sequence for high potency of expressing heterologous protein (Fig. 6C).

After G418-resistant stable cell lines were picked up, a semiquantitative RT-PCR analysis was performed to determine the HTA mRNA expression in different clones. QSG-7701-HTA-2, the clone with the highest HTA expression, was chosen to perform subsequent in vitro experiments (Fig. 7).

Overexpression of HTA promotes cell growth in vitro. The rate of the cell proliferation in the clone QSG-7701-HTA-2 was significantly increased compared to QSG-7701-c. Cell proliferation curve detected by CCK-8 assay (Fig. 8) and colony formation assay (Fig. 9) showed that the overexpression of HTA upregulated the cell proliferation and colony formation ability of QSG-7701 cells. The cell doubling time of QSG-7701-HTA-2 was $3.87 \pm 0.187$ days, while QSG-7701-c was $9.14 \pm 0.143$ days. Cell cycle distribution detected by flow cytometry is shown in Fig. 10. This result is in line with the above results. 
A

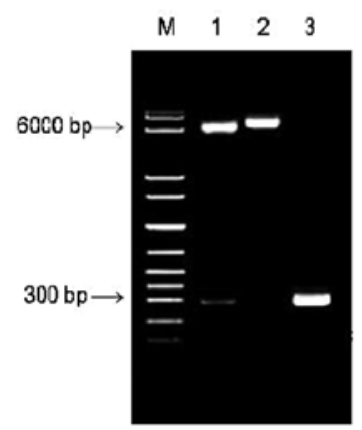

B

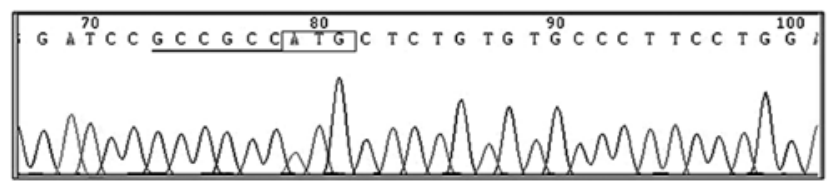

C

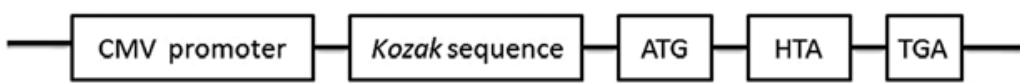

Figure 6. Construction of plasmid pcDNA3.1(+)-HTA. (A) Identification of recombinant plasmid pcDNA3.1(+)-HTA by double enzyme digestion and bacterial colony PCR. Lane M, DNA ladder; lane 1, double digestion with BamHI and EcoRI; lane 2, pcDNA3.1 (+)-HTA without digestion; lane 3, PCR product of positive bacterial colony. (B) Part sequence of the 5' terminal of pcDNA3.1(+)-HTA plasmid. The square shows the initial codon of $H T A$. (C) The schematic diagram of recombinant prokaryotic expression plasmid pcDNA3.1(+)-HTA.

$\mathbf{A}$

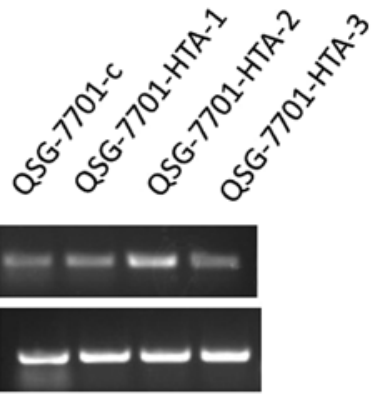

B

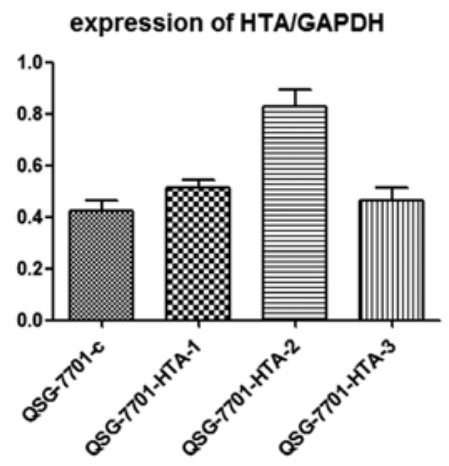

Figure 7. Determination of the HTA mRNA expression in different QSG-7701 clones. (A) The mRNA expression of HTA and GAPDH detected by RT-PCR. (B) Expression ratio of $H T A / G A P D H$ evaluated by gray scan.

\section{cell proliferation curve}

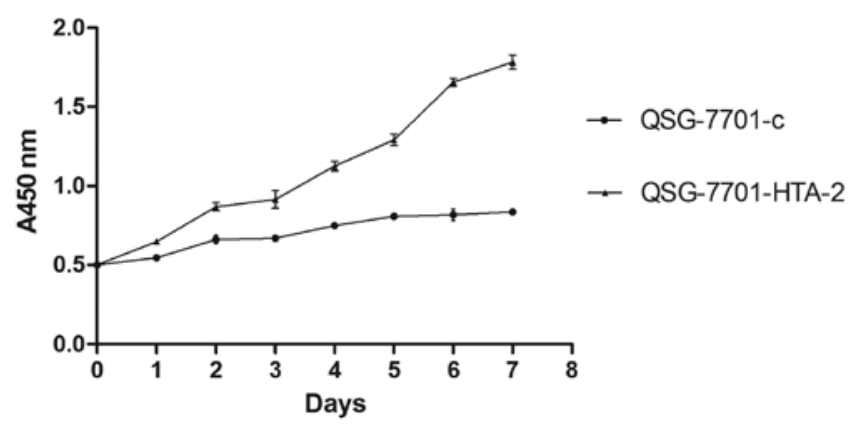

Figure 8. Cell proliferation curve of QSG-7701-c and QSG-7701-HTA-2 detected by CCK-8 assay.

\section{Discussion}

HTA is a novel tumor associated gene screened by our research group. To date, only a few studies have focused on the HTA gene. Specific expression characteristics and the cancer-promoting effect revealed by knockdown experiment suggested that HTA may play a role in HCC development (10). In order to thoroughly explore the role of $H T A$, the full length sequence of HTA is imperative for the further investigations such as overexpression and functional clarification. In this study, the full length cDNA of HTA was obtained by 3'-RACE and 5'-RACE; HTA mRNA expression in different hepatic cell lines and normal cell lines was analyzed by northern blot assay, and the overexpression of HTA cDNA in the hepatic cell line was performed to clarify the biological function of the HTA gene.

There were certain difficulties in obtaining the full length cDNA sequence of the HTA gene, due to its relatively low expression even in the HepG2 cell line, which is considered to have the highest expression of the HTA gene. The smart technology, which is considered the most advanced approach to obtain the full length cDNA of a certain gene with a partial known sequence, was chosen for the full length cDNA of the HTA gene (11). At the same time, hot start PCR polymerase was applied to avoid non-specific amplification in lower temperatures (14) and a touch-down PCR program was applied to avoid non-specific amplification in early cycles and to improve the efficiency of specific amplification in later cycles of the program (15).

The accuracy and integrity of the cDNA sequence obtained by $3^{\prime} / 5^{\prime}-\mathrm{RACE}$ require further confirmation. Following $3^{\prime} / 5^{\prime}-$ RACE, RT-PCR was performed. The sequence of HTA cDNA was amplified by full length primer. As the HTA gene is unique in quadrumana and exclusively found in the genome of humans and anthropoids, we could not confirm its full length cDNA sequence by homologous alignment (16). Therefore, the full length of the HTA gene according to the disciplines as following: the ready frame of the gene was integral: there was a terminal codon in the same frame in the upstream of the ORF's initial codon ATG and there was polyA tail. At the same time, it was proved that the length of HTA transcripts defined by northern blot analysis was consistent with the sequence obtained by RACE. 
A

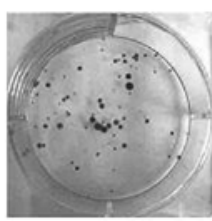

QSG-7701-c

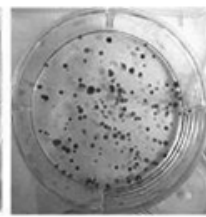

QSG-7701-HTA-2
B colony formation assay

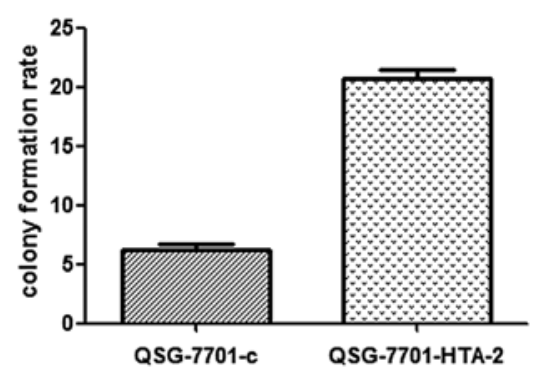

Figure 9. Colony formation assay. (A) The colony formation assay of QSG-7701-c and QSG-7701-HTA-2 cell lines. (B) The colony formation rate of QSG-7701-c and QSG-7701-HTA-2.

A
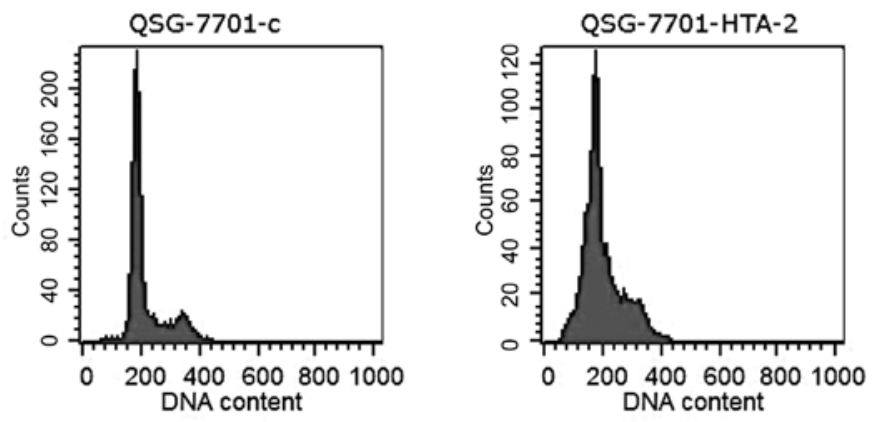

B

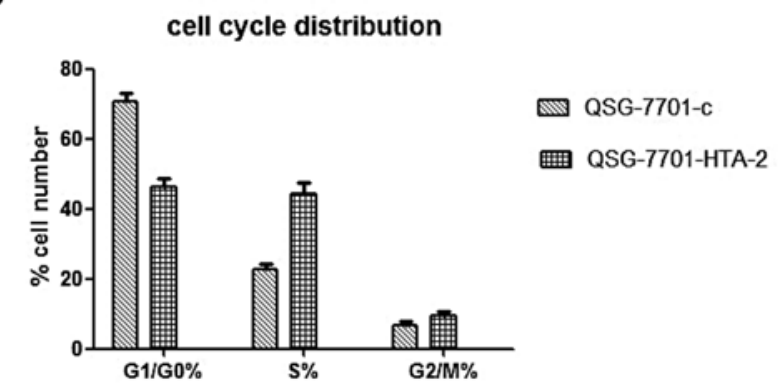

Figure 10. Cell cycle distribution. (A) Cell cycle distribution of QSG-7701-c and QSG-7701-HTA-2 detected by flow cytometry. (B) Cell cycle distribution percent of QSG-7701-c and QSG-7701-HTA-2.

Alternative splicing occurs as a normal phenomenon in eukaryotes, where it greatly increases the biodiversity of proteins that can be encoded by the genome $(17,18)$. In humans, approximately $95 \%$ of multi-exonic genes are alternatively spliced (18). There are numerous modes of alternative splicing observed, of which the most common is exon skipping (17). It was reported that alternative splicing plays a crucial role in receptor diversity generation and growth and development regulation $(19,20)$, particularly reflected in the nervous $(21,22)$ and the immune system $(23,24)$, which was closely related to their function diversity and reaction sensibility. It was estimated that $15 \%$ of disease occurred mutations would affect the splicing of pre-mRNA $(25,26)$. We clarified that the second introns of the HTA gene were selectively retained in mRNA and produced a small quantity of longer transcript. Whether this alternative splicing plays a role in carcinogenesis requires further elucidation.

In vitro experiments revealed that overexpression of the HTA gene in the hepatic cell line QSG-7701 promotes the proliferation and colony formation ability of the cells, which proved the carcinogenesis by the HTA gene, and it was consistent with the interference experiment in a previous study (10). The result confirmed that the HTA gene plays an important role in HCC development.

In conclusion, this study obtained and identified the full length cDNA sequence of the HTA gene and analyzed its alternative splicing form, further elucidating the structure of the HTA gene and providing the foundations for HTA gene function studies. Primary function study of the HTA gene by overexpression in vitro proved its role in $\mathrm{HCC}$ carcinogenesis.

\section{Acknowledgements}

This study was supported by the National Natural Science Foundation of China (81201903 and 2010CB833605), the Science and Technology Department Research Foundation of Hunan Province (2010SK3132), and the PhD Candidates' Research Innovation Project of Hunan Province (CX2011B050), China. 


\section{References}

1. El-Serag HB: Hepatocellular carcinoma. N Engl J Med 365 1118-1127, 2011.

2. Siegel R, Naishadham D and Jemal A: Cancer statistics, 2012. CA Cancer J Clin 62: 10-29, 2012.

3. DuBray BJ, Chapman WC and Anderson CD: Hepatocellular carcinoma: a review of the surgical approaches to management. Mo Med 108: 195-198, 2011.

4. Jia HL, Ye QH and Qin LX, et al: Gene expression profiling reveals potential biomarkers of human hepatocellular carcinoma. Clin Cancer Res 13: 1133-1139, 2007.

5. Keng VW, Villanueva A, Chiang DY, et al: A conditional transposon-based insertional mutagenesis screen for genes associated with mouse hepatocellular carcinoma. Nat Biotechnol 27: 264-274, 2009.

6. Zender L, Xue W, Zuber J, et al: An oncogenomics-based in vivo RNAi screen identifies tumor suppressors in liver cancer. Cell 135: 852-864, 2008

7. Pei Y, Zhang T, Renault V and Zhang X: An overview of hepatocellular carcinoma study by omics-based methods. Acta Biochim Biophys Sin (Shanghai) 41: 1-15, 2009.

8. Villanueva A, Minguez B, Forner A, Reig M and Llovet JM: Hepatocellular carcinoma: novel molecular approaches for diagnosis, prognosis, and therapy. Annu Rev Med 61: 317-328, 2010.

9. Zender L, Villanueva A, Tovar V, Sia D, Chiang DY and Llovet JM: Cancer gene discovery in hepatocellular carcinoma. J Hepatol 52: 921-929, 2010.

10. Liu Y, Li Y, Guo F, et al: Identification of HTA as a novel-specific marker for human hepatocellular carcinoma. J Cancer Res Clin Oncol 136: 1187-1192, 2010.

11. Scotto-Lavino E, Du G and Frohman MA: 5' end cDNA amplification using classic RACE. Nat Protoc 1: 2555-2562, 2006.

12. Scotto-Lavino E, Du G and Frohman MA: 3' end cDNA amplification using classic RACE. Nat Protoc 1: 2742-2745, 2006.

13. Kozak M: Structural features in eukaryotic mRNAs that modulate the initiation of translation. J Biol Chem 266: 19867-19870, 1991.
14. Paul N, Shum J and Le T: Hot start PCR. Methods Mol Biol 630: 301-318, 2010.

15. Pratyush DD, Tiwari S, Kumar A and Singh SK: A new approach to touch down method using betaine as co-solvent for increased specificity and intensity of GC rich gene amplification. Gene 497: 269-272, 2012.

16. Loytynoja A: Alignment methods: strategies, challenges, benchmarking, and comparative overview. Methods Mol Biol 855: 203-235, 2012.

17. Black DL: Mechanisms of alternative pre-messenger RNA splicing. Annu Rev Biochem 72: 291-336, 2003.

18. Pan Q, Shai O, Lee LJ, Frey BJ and Blencowe BJ: Deep surveying of alternative splicing complexity in the human transcriptome by high-throughput sequencing. Nat Genet 40: 1413-1415, 2008.

19. Roberts GC and Smith CW: Alternative splicing: combinatorial output from the genome. Curr Opin Chem Biol 6: 375-383, 2002.

20. Kelemen O, Convertini P, Zhang Z, et al: Function of alternative splicing. Gene: Aug 15, 2012 (Epub ahead of print).

21. Norris AD and Calarco JA: Emerging roles of alternative pre-mRNA splicing regulation in neuronal development and function. Front Neurosci 6: 122, 2012.

22. Dever SM, Xu R, Fitting S, Knapp PE and Hauser KF: Differential expression and HIV-1 regulation of $\mu$-opioid receptor splice variants across human central nervous system cell types. J Neurovirol 18: 181-190, 2012.

23. Magg T, Mannert J, Ellwart JW, Schmid I and Albert MH: Subcellular localization of FOXP3 in human regulatory and nonregulatory T cells. Eur J Immunol 42: 1627-1638, 2012.

24. Martinez NM, Pan Q, Cole BS, et al: Alternative splicing networks regulated by signaling in human T cells. RNA 18: 1029-1040, 2012.

25. Liu J, Lee W, Jiang Z, et al: Genome and transcriptome sequencing of lung cancers reveal diverse mutational and splicing events. Genome Res: Nov 1, 2012 (Epub ahead of print).

26. Singh RK and Cooper TA: Pre-mRNA splicing in disease and therapeutics. Trends Mol Med 18: 472-482, 2012. 